\title{
La red vial como estructura posibilitante del desarrollo en el área metropolitana de Córdoba, Argentina
}

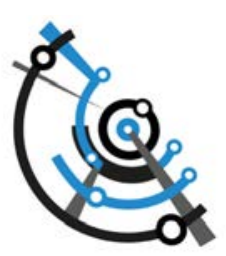

\author{
Sara María Boccolini \\ Centro de Investigaciones y Estudios sobre Cultura y Sociedad - CONICET/UNC \\ https://orcid.org/oooo-0002-2864-8744
}

Recibido: 7 de junio de 2019. Aceptado: 29 de agosto de 2019.

\begin{abstract}
Resumen
La red vial de una región urbana funciona como canal de flujos (de personas y bienes), conduciéndolos desde y hacia distintos puntos en el territorio, pero también forzando este tráfico en ciertas regiones en desmedro de otras. Debido a esto, su análisis es indispensable para comprender el escenario actual de una región metropolitana y delinear tendencias de desarrollo a mediano plazo. Sin embargo, debido a su extensión y complejidad, su estudio suele priorizar valoraciones cualitativas. En este trabajo se analizó la red vial del área metropolitana de Córdoba (Argentina) dentro del marco instrumental y conceptual de la Sintaxis Espacial, que permite valorar cuantitativamente los niveles de conectividad e integración global de los elementos que componen la red en función de su articulación dentro del sistema vial y sus características topológicas. Además, se analizó la Sintaxis Espacial tanto en función de su potencial como herramienta analítica como de las limitaciones en su capacidad explicativa. Para superar éstas últimas, los resultados se analizaron en combinación con patrones de distribución de la población y de equipamientos y servicios urbanos a escala metropolitana, y se determinaron tendencias de desarrollo en cuanto a reestructuración del área urbanizada y polarización socioeconómica.
\end{abstract}

Palabras clave: Sintaxis espacial. Conectividad. Integración. Red vial. Región metropolitana.

\section{The Road Network as a Development-Enabling Structure in Metropolitan Córdoba, Argentina}

\begin{abstract}
The road network of an urban region works as a channel of flows (both of people and comodities), driving them to and from certain territories, but also driving this traffic to certain regions to the detriment of others. Therefore, its analysis is essential to understand the current scenario of a metropolitan region, as for outlining medium-term trends. However, due to metropolitan networks' length and complexity, its study usually
\end{abstract}


prioritizes qualitative assessments. Instead, we analyzed here the road network of the metropolitan Córdoba (Argentina); we did so within the instrumental and conceptual framework of Spatial Syntax. The Spatial Syntax approach allows us to quantitatively assess the levels of connectivity and global integration of the elements that integrate the road network, according to their articulation within the road system and its topology. At the same time, we discussed both the potential and the limitations of Spatial Syntax as an analytical tool; in order to overcome the latter, we analyzed the results of Space Syntax analysis in combination with patterns of population distribution, as much as urban facilities and services distribution, determining development trends in terms of urban area restructuration and socioeconomic polarization.

Keywords: Space syntax. Conectivity. Integration. Road network. Metropolitan region.

Palavras chave: Sintaxe espacial. Conectividade. Integração. Rede rodoviária. Região Metropolitana.

\section{Introducción}

El trazado vial en una aglomeración urbana es resultado de condicionantes geográficos y procesos históricos, a la vez que estructura los espacios públicos de la ciudad y condiciona, en gran medida, el modo en el cual edificaciones e infraestructuras se interrelacionan en el tejido urbano. Al establecer barreras y conducir las movilidades de agentes y recursos, y creando ubicaciones diferenciadas, también condiciona la localización de usos, actividades y estratos socioeconómicos. Además, como la mayor parte de los elementos construidos en un sistema urbano, tiene una gran inercia frente a los cambios, a comparación de elementos dinámicos como los agentes humanos, bienes móviles, localización de actividades, etc.: la red vial es una de las partes más permanentes de su estructura física. Por lo tanto,

Estudiar su proceso de formación, así como sus implicaciones en el contexto urbano, constituye una condición fundamental para el reconocimiento de las potencialidades y limitaciones de nuestras ciudades contemporáneas (Tibiriçá de Saboya et al., 2016:131, traducción propia).

En este trabajo se aborda el análisis del sistema vial del área metropolitana de Córdoba (AMCBA) desde el marco metodológico de la sintaxis espacial (SE). La SE es un conjunto de técnicas para analizar diseños espaciales y patrones de actividad humana en edificios y regiones urbanas. Fue desarrollada en la década de 1970 por Bill Hillier, Julienne Hanson y sus colegas en The Bartlett School of Architecture (University College de Londres), donde continúan desarrollando teórica y metodológicamente esta herramienta (Hillier, 2015; Hillier y Julienne, 2014; Hanson, 2003). Su Space Syntax Laboratory (spacesyntax.net) brinda los fundamentos en que se apoya el análisis desarrollado en este trabajo.

La SE se basa en varias premisas generales: la más desarrollada por el Space Syntax Laboratory fundamenta sus conclusiones en la producción social del espacio: “...se parte del entendimiento de que la forma urbana es tanto resultado de estructuras sociales como condicionante de procesos de producción y reproducción social" (Tibiriçá de Saboya et al., 2016:132, traducción propia).

Según esta premisa, el espacio no es un mero escenario para las actividades humanas, sino que, a través del estudio del espacio pueden explicarse y predecirse pautas de comportamiento de los agentes que interactúan en él. Los trabajos desarrollados en esta línea avanzan en el supuesto potencial de la SE para predecir el comportamiento humano y en consecuencia los movimientos a través de una red espacial, ya sea a pequeña, mediana o gran escala. 
Esta base determinista que se concentra en el espacio público para predecir el sistema de movimientos en un sistema urbano ha suscitado controversia sobre la fiabilidad de esta hipótesis. En efecto, es discutible la postura excluyente de una red de espacios como contenedor abstracto de flujos, que relativiza la importancia de factores como la topografía, el diseño de la envolvente espacial, el caudal de tráfico y la capacidad de la red para sostenerlo, la densidad de edificación y de ocupación, y las estructuras políticoculturales y productivas de la comunidad para definir el modo en que se produce el espacio (Batty, 2001). Acordando además con Carlo Ratti (2004), el marco instrumental de la SE no aborda los sesgos socio-culturales de los agentes que se movilizan en el sistema, ni las barreras culturales o ambientales.

Más allá de lo anterior, el marco conceptual y analítico de la SE también avanza sobre el concepto de espacio urbano como una red espacial que funciona como posibilitante, a través de la cual se produce el flujo de personas y bienes: lo que sucede en cualquier espacio individual (un corredor, una calle o un espacio público) está influenciado fundamentalmente por las relaciones entre ese espacio y la red de espacios a los que está conectado (Hillier, 2015:112). Esto implica que

(...) cada espacio de una ciudad es lo que es, en gran parte, por el papel que desempeña en el contexto más amplio, por las relaciones que establece con sus vecinos inmediatos y distantes, por la posición central o periférica que ocupa, por estar más o menos en el paso entre otros lugares (Tibiriçá de Saboya et al., 2016:137, traducción propia).

Por lo tanto, este trabajo explora el potencial analítico de la SE para analizar la red de espacios de circulación vial pública y valorarla como posibilitante o limitante de flujos. El análisis se realiza en función de la cuantificación de indicadores que explican estas propiedades: la integración y la conectividad de cada uno de los elementos que componen el sistema -en este caso, vectores que representan las vías de la red. Estos indicadores son esenciales para reconstruir la capacidad de la red vial como sistema posibilitante de flujos de personas y bienes:

》 La integración hace referencia al grado en que un nodo se integra al sistema (integración global) o a parte del sistema -un distrito o barrio- (integración local). La integración mide la distancia topológica de un elemento a todos los otros elementos que conforman el sistema (Tagliari, 2018). Esto quiere decir que la integración de un elemento será mayor cuando sea necesario conectarse a una menor cantidad de elementos para llegar a todos y cada uno de los otros elementos que conforman el sistema.

" La conectividad refiere al grado en que una vía está conectada a las demás en el sistema; la métrica de conectividad mide el grado en que una vía controla el acceso a sus vecinas más cercanas (Telega, 2016), es decir, que la conectividad de un elemento será mayor cuando mayor sea la cantidad de elementos del sistema a los que está vinculado directamente (Tagliari, 2018).

Según estas definiciones, puede decirse que, mientras la primera mide la facilidad de acceso, la segunda mide el flujo posible a través de cada vector del sistema con base en la topología de la red.

Para realizar el análisis cuantitativo de los valores de estos indicadores en los elementos que componen un sistema espacial, la SE recurre a modelos espaciales que representan el sistema con elementos discretos simples. Estos elementos pueden derivarse geométricamente (por ejemplo, puntos, líneas axiales, segmentos, espacios convexos, isovistas) o definirse funcionalmente (por ejemplo, habitaciones en edificios), en función de las interrelaciones que estadísticamente pueden llevarse a cabo 
en función de su estructura topológica. En consecuencia, la SE abarca numerosos procedimientos para modelar y analizar los sistemas espaciales; entre ellos están los mapas axiales y los mapas de segmentos (los más difundidos en estudios de redes viales), los mapas convexos, los gráficos de visibilidad, las isovistas, y los modelos de agentes, de usos del suelo, de edificaciones, de eventos, etc. En este trabajo se adopta la premisa propuesta en Tagliari (2018), Stavroulaki, Marcus, Berghauser Pont y Staffan Nilsson (2017), Scheel, Mann y Pavié (2017) y en Green y Mora (2005) -entre otros- donde el sistema vial se modela como un mapa axial, que representa las vías como vectores georreferenciados según su eje medio, comunicados entre sí por nodos para formar la red a escala metropolitana.

La utilización de mapas axiales de ejes de vías significa una gran ventaja para el desarrollo y difusión de la metodología analítica de la SE aplicada a escala urbana y regional: permiten el uso de mapas de vectores basados en modelos SIG utilizados en la mayoría de los trabajos de modelado urbano, y han demostrado ser alternativas válidas al mapa axial inicialmente propuesto por el Space Syntax Laboratory (Stavroulaki et al., 2017).

Por lo tanto, y estableciendo entonces las limitaciones en cuanto a la capacidad interpretativa del análisis en el marco de la SE, en este trabajo se analiza la red vial del AMCBA para cuantificar la integración global y la conectividad en los distintos puntos del territorio metropolitano con base a la red vial y focalizándose -debido a la escala- en el flujo de vehículos y no de peatones.

Inicialmente, el trabajo caracteriza brevemente el AMCBA como sistema urbano y regional. Luego, detalla el proceso de elaboración, verificación y análisis del mapa axial de ejes de vías dentro del marco analítico de la SE, para calcular los valores de integración global y conectividad de cada uno de los vectores. Seguidamente, discute críticamente los resultados en función de un análisis comparativo con los patrones de distribución poblacional (tanto la distribución cuantitativa como según su nivel socioeconómico) y de distribución de equipamientos y servicios urbanos (EySU) en el territorio, determinando el rol de la red vial como posibilitante o limitante del acceso de la población a las áreas de mejor calidad urbana y ambiental.

Los principales resultados muestran una red altamente concentrada en Córdoba, el aglomerado urbano principal, y con escasa conectividad en áreas periurbanas y rurales. Y aunque la topografía es un factor de importancia que limita la conectividad e integración de parte de la región, las estructuras productivas y políticas que definen el proceso de urbanización también conducen a producir una red fragmentada, con bajos niveles de conectividad incluso entre sectores muy cercanos de la red. Esto no sólo influye en la escasa conexión entre sectores urbanos contiguos; aunque la mayor parte de la población metropolitana habita en áreas de alta conectividad e integración en el sistema, la población rural -especialmente la población de menor nivel socioeconómico (NSE) que se encuentra más segregada- reside en áreas de escasa conectividad con áreas urbanas cercanas, dependiendo fuertemente del acceso a las alejadas áreas urbanas centrales que concentran los EySU.

A lo anterior se suma una tendencia de redistribución poblacional desde las áreas mejor conectadas e integradas de la región hacia sectores con menores niveles de integración y conectividad, que difícilmente puedan elevarse a mediano plazo. Esta redistribución poblacional, sumada a tendencias de polarización socioeconómica y la concentración territorial de EySU muestran además una tendencia de concentración de la población de mayor NSE en las áreas de mejor calidad ambiental y urbana y gran accesibilidad a EySU, mientras que la población de menores recursos tiende a ubicarse en áreas de escasa accesibilidad a EySU. 
Si bien en este trabajo no se plantea la SE como un instrumento predictivo, es posible verificar el potencial de la SE para profundizar análisis y diagnósticos urbano-regionales. Los resultados apoyan la premisa de que cartografiar la integración o conectividad de la red vial constituye una labor esencial para comprender la estructura urbana de la región y sus principales tendencias de desarrollo actual y a mediano plazo.

\section{Una constelación de ciudades entre la llanura y las sierras}

El AMCBA se ubica en la región central de Argentina, extendiéndose desde la llanura pampeana al Este y la zona de piedemonte serrano, hacia el cordón de las Sierras Chicas al Oeste y más allá, a lo largo de los valles de las Sierras Chicas (Noroeste) y de Punilla (Oeste). La región estudiada en este trabajo se basa en la delimitación del AMCBA propuesta en IPLAM (2017) e Irós, Moiso, Bravo, Alonso y Gómez (2012) (Figuras 1 y 2). ${ }^{1}$

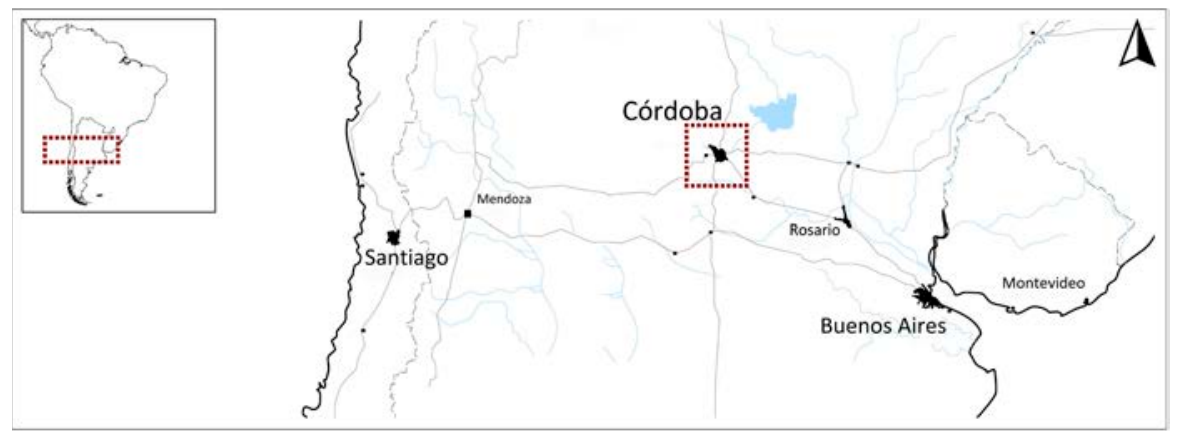

Figura 1. Ubicación relativa del AMCBA en la región central del cono Sur de América Latina. Fuente: elaboración propia (2015) con base en datos de IDERA.

La aglomeración urbana principal del AMCBA es la ciudad de Córdoba, principal centro de EySU de la región. Está conectada regionalmente a través de una red de rutas y caminos que se extiende radialmente hacia los principales centros urbanos del país. A lo largo de esa red principal se encuentran los aglomerados urbanos medios y pequeños del AMCBA, que funciona, así, como un sistema urbano metropolitano en red extendido en casi $8.000 \mathrm{~km}^{2}$ (Figura 2). 


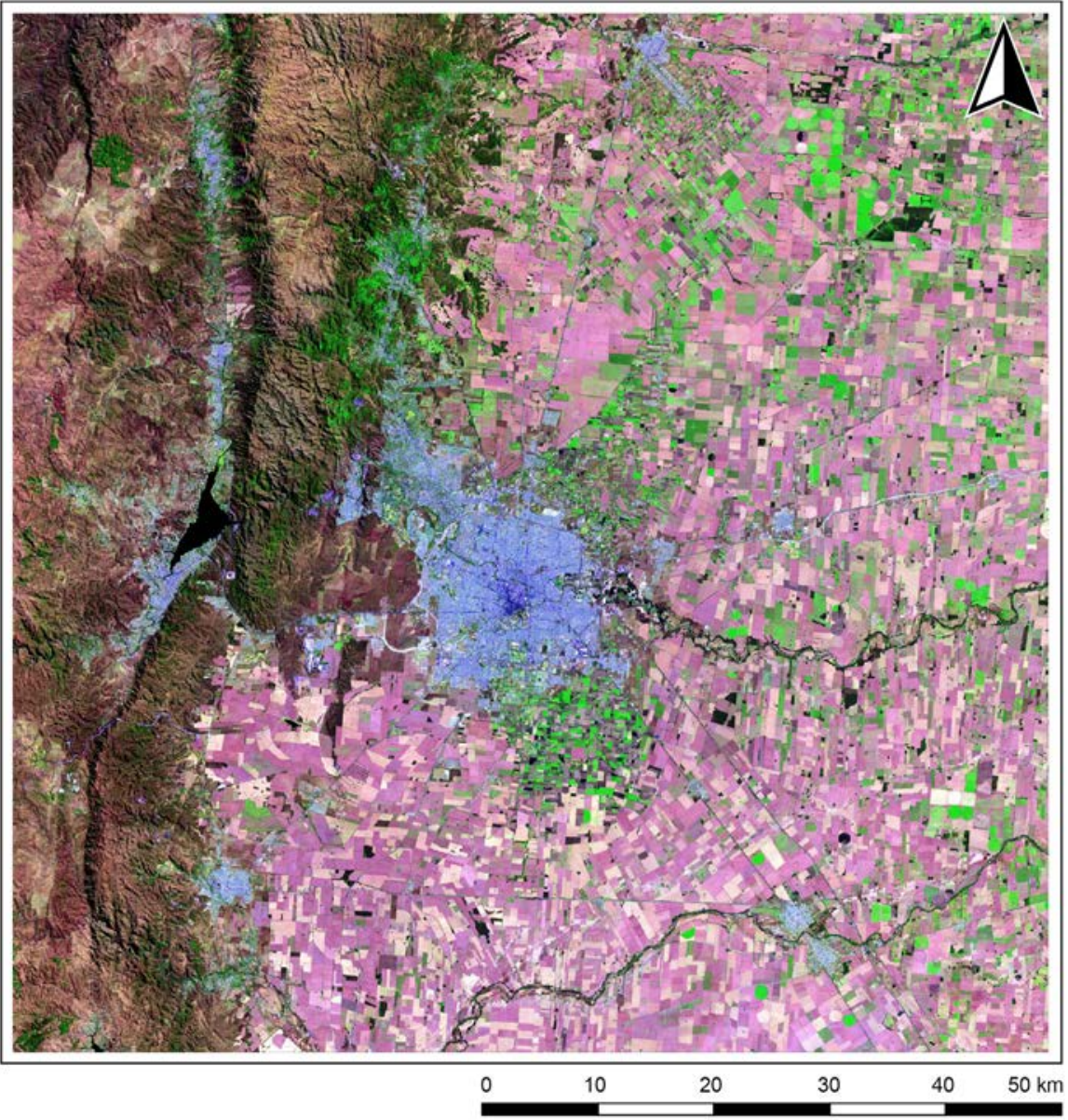

Figura 2. AMCBA. Imagen satelital. Imagen LANSAT 8 del 8 junio de 2018 procesada en combinación de bandas 6-5-2. Los tonos violáceos indican la superficie edificada; los tonos rosados indican superficie ocupada con cultivos en secano; los tonos verdes indican el área con cultivo bajo riego (principalmente hortícola); los tonos ocres indican el área ocupada por monte serrano natural. El color negro indica superficie ocupada con cuerpos de agua: al Oeste (en el Valle de Punilla) se identifica el Dique San Roque, desde el que corre el río Suquía hacia el Este, atravesando la ciudad de Córdoba -la mayor área urbanizada en la figura. Fuente: Elaboración propia (2019) procesando con QGIS una imagen LANSAT 8 obtenida en el repositorio digital de United States Geological Survey (https://earthexplorer.usgs.gov).

Desde Córdoba y hacia la llanura pampeana al Este, los centros urbanos albergan principalmente EySU para las actividades agropecuarias de tipo extensivo que predominan en ese sector (con predominio de cultivo de oleaginosas y cereales). Los centros urbanos que se extienden en la zona serrana articulan equipamientos y servicios orientados principalmente al turismo serrano y, en menor medida, actividades agropecuarias de mediana y pequeña escala. Las actividades industriales tienden a concentrarse en la periferia de los mayores aglomerados urbanos, principalmente al Sur, Sureste, Este y Noreste de la ciudad de Córdoba.

Los flujos de red vial metropolitana están caracterizados tanto por los movimientos a escala nacional -sobre todo como nodo de conexión de la región Norte y Oeste del país desde y hacia los puertos de Buenos Aires y Rosario-, como por los movimientos pendulares entre la ciudad de Córdoba y los municipios menores o áreas rurales por la oferta de EySU concentrada en Córdoba (Peralta y Liborio, 2014a; Tecco, 1999).

El complejo proceso de urbanización de la región ha sido estudiado ya en numerosas ocasiones (UVITEC e Innovos Group SA, 2017; ADEC, 2014, 2015a, 2015b; Irós et al., 
2012; Tecco, 1999; Dirección de Planeamiento urbano - Secretaría de Planeamiento Urbano - Dirección de Hábitat, 1981). Según plantean estos estudios, en el AMCBA se combina la tendencia de concentración prácticamente macrocefálica de población y EySU en Córdoba con la tendencia de crecimiento por extensión -más o menos planificada- a lo largo de las vías principales de la red, en forma de urbanizaciones con distinto grado de conexión a la red existente. En los aglomerados de mayor y más constante crecimiento, estas urbanizaciones recientes se han conurbado con el área urbana más antigua de cada aglomeración, formando un territorio urbanizado continuo - pero de muy baja densidad de ocupación- que se extiende cada vez más, conurbando incluso aglomerados medianos y pequeños entre sí (Figura 3). En otras ocasiones, el crecimiento por extensión urbaniza áreas con muy poca integración a la trama urbana existente, dependiendo de la red vial principal para integrarse a la red urbana (Figura 4).

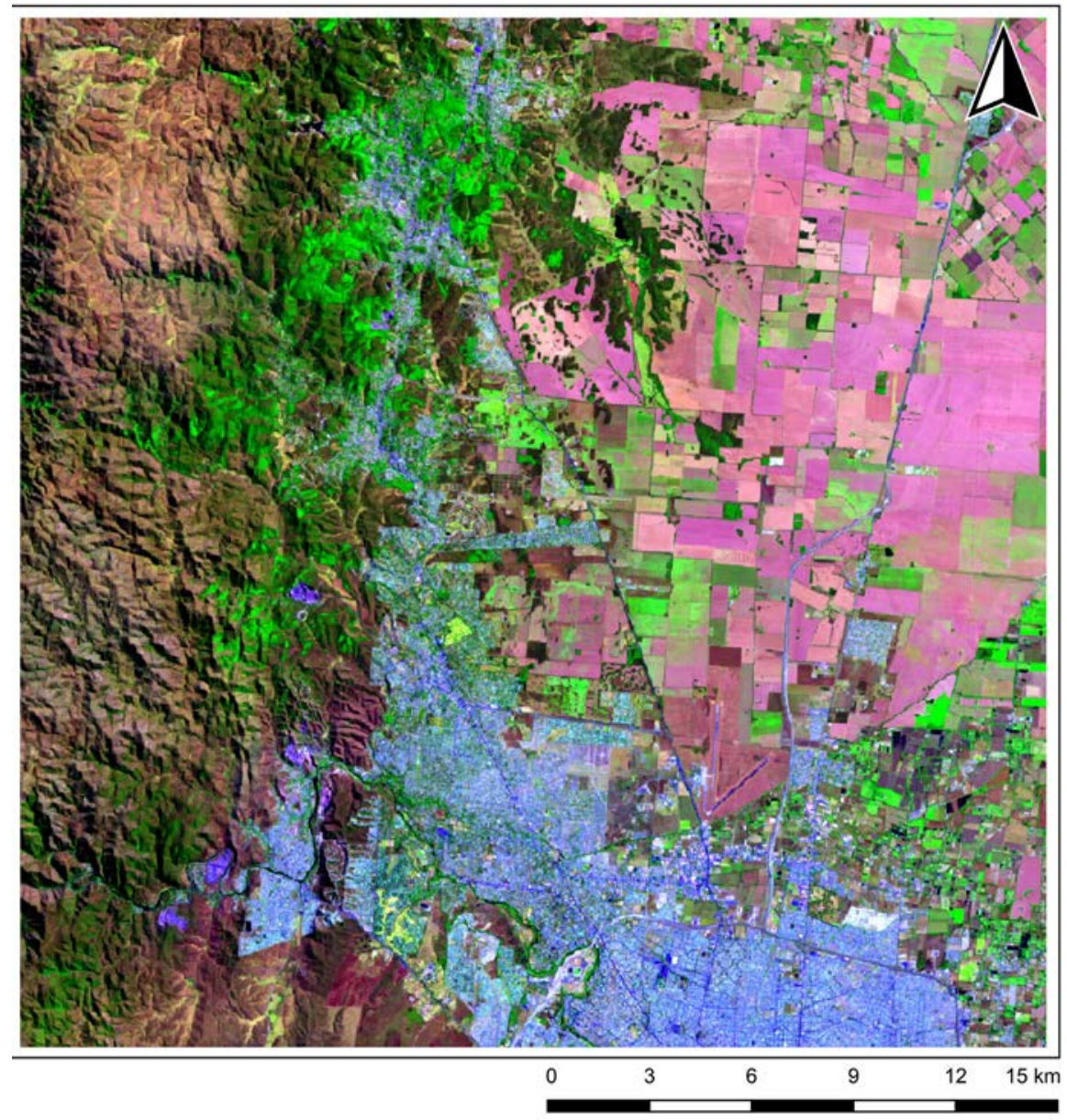

Figura 3. Imagen satelital del AMCBA (detalle). Imagen LANSAT 8 del 8 junio de 2018 procesada en combinación de bandas 6-5-2. Sector Noroeste de Córdoba (derecha, abajo) yáreas conurbadas de Villa Allende, Saldán, Unquillo y Mendiolaza al Noroeste, en el Valle de las Sierras Chicas (centro y arriba). Fuente: elaboración propia (2019) procesando una imagen obtenida en el repositorio digital de United States Geological Survey (https://earthexplorer.usgs.gov). 


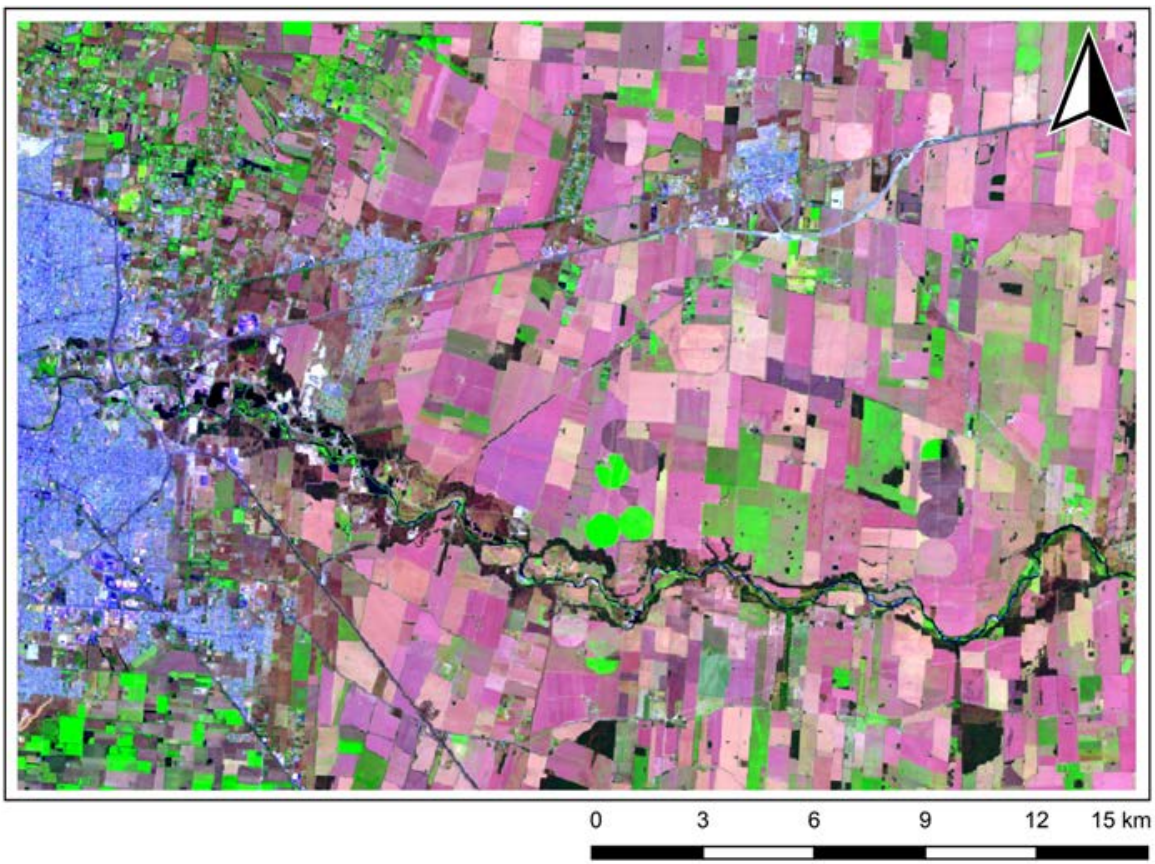

Figura 4. Imagen satelital del AMCBA (detalle). Imagen LANSAT 8 del 8 junio de 2018 procesada en combinación de bandas 6-5-2. Sector Este de Córdoba (izquierda) yáreas urbanizadas de Malvinas Argentinas y Montecristo hacia el Este (centro y derecha). Fuente: Elaboración propia (2019) procesando una imagen obtenida en el repositorio digital de United States Geological Survey (https://earthexplorer.usgs.gov).

Junto a las estructuras de explotación y producción a escala regional, la topografía es una condición primordial que define el trazado vial en cada región, y el AMCBA no es una excepción. En los aglomerados del sector serrano al Oeste predominan trazados de ejes curvos e irregulares, con numerosas discontinuidades en la red. Al contrario, en la llanura del sector Este predominan los trazados reticulares más o menos regulares y con mayor continuidad en la trama interna de cada aglomerado (Figuras 3 y 4 ; ver además más adelante las Figuras 6 y 7). Los pequeños cursos de agua (más numerosos y de cauces sumamente irregulares en la región serrana), combinados con el sistema de canales de riego en la llanura pampeana) y la red ferroviaria contribuyen a fragmentar el trazado de la red vial en el territorio.

Un territorio de tal extensión y complejidad como el AMCBA está estructurado por una red vial igualmente compleja y extensa. A continuación, se desarrolla el proceso seguido para reconstruir y analizar esa red vial en el marco de la SE.

\section{Reconstrucción del mapa axial y análisis de conectividad e integración global}

Una vez definido el marco conceptual e instrumental en que se encuadra este trabajo y caracterizado el territorio analizado, se reconstruye el mapa axial de la red vial del AMCBA. Para ello se parte de la compilación de mapas de ejes de vías de los municipios del AMCBA que están disponibles para su descarga en el geoportal web de la Dirección Provincial de Estadística y Censos de Córdoba (DEyC). Cada uno de estos mapas axiales se revisa y corrige individualmente, ya que algunos cursos de agua y límites de propiedad figuran como vectores de la red vial, o vías de ancho mayor al promedio (avenidas, boulevares) están representadas por dos ejes paralelos en lugar de uno. En esta etapa se constata además que, al basarse en datos del año 2010, los trazados urbanos más recientes no están incluidos. Estos trazados faltantes, así como 
el relevamiento de la red interurbana de rutas y caminos se completan manualmente con base en el relevamiento realizado por el Instituto de Planificación del Área Metropolitana (IPLAM) compilado por el equipo del proyecto Bases ambientales para el ordenamiento territorial del espacio rural de la provincia de Córdoba (BAOTCba).

El relevamiento manual -a pesar de su poca eficiencia en cuanto a uso de recursos humanos y tiempo de trazado necesario- resulta en un mapa axial confiable en cuanto a fidelidad a la red vial existente, y con menores errores de dibujo que los mapas obtenidos mediante procesamiento automatizado (que resultan en poca economía de vectores -sobre todo en trazados irregulares-, vectores duplicados, errores en las intersecciones en nodos, islas de vectores o vectores huérfanos). En efecto, la etapa de verificación del modelo es mucho menos laboriosa para el modelo trazado manualmente.

El mapa axial de ejes de vías obtenido finalmente se muestra en la figura 5. Este mapa permite analizar en detalle las características de la red vial urbana e interurbana de la zona pampeana del Este (predominio de trazado urbano reticular e interurbano de líneas rectas) y las conurbaciones con la ciudad de Córdoba al Noroeste (de trazado irregular y discontinuo debido principalmente a la topografía). Esto se constata en las Figuras 6 y 7, que muestran detalles del mapa axial coincidentes con los sectores mostrados en las Figuras 3 y 4 respectivamente.

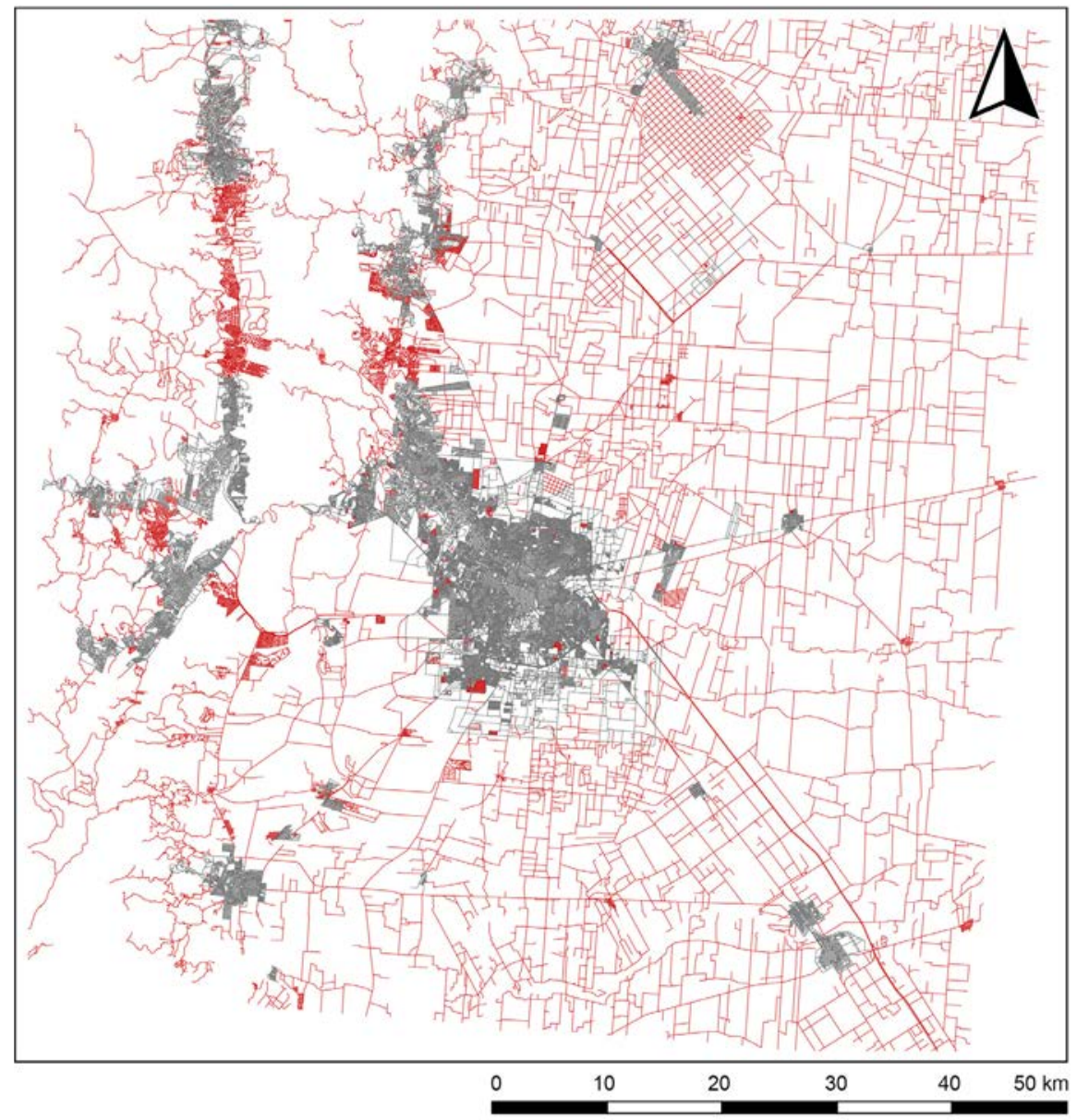

Figura 5. AMCBA. Red vial (2015). Mapa axial de ejes de vías. En gris se muestran los vectores recopilados de bases de datos de la DEyC; en rojo se indican los vectores trazados manualmente con base en datos del IPLAM. Fuente: elaboración propia (2019) con base en datos de IDERA, DEyC, IPLAM y BAOTCba. 


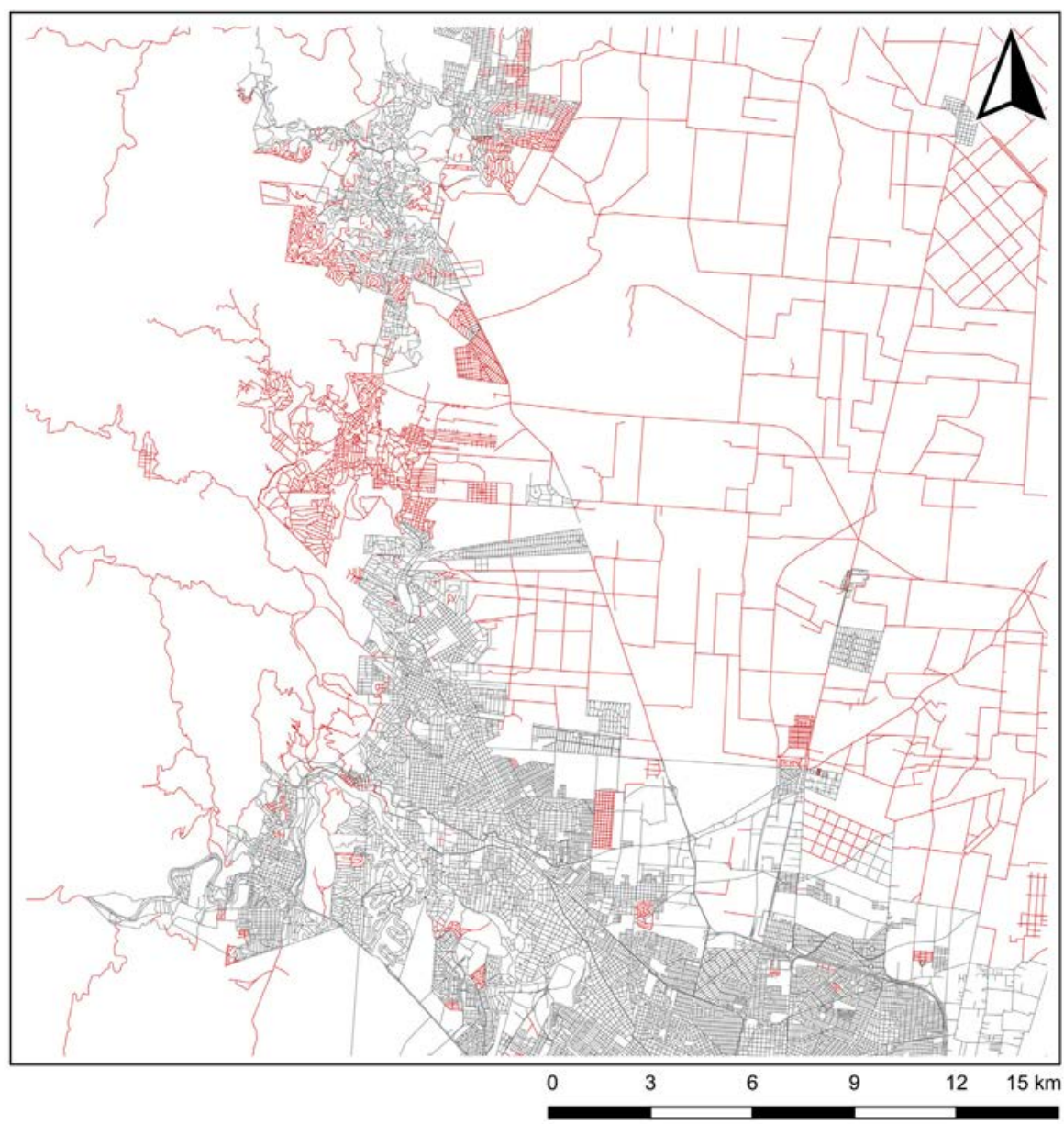

Figura 6. AMCBA. Red vial (2015) (detalle). Sector Noroeste de Córdoba (derecha) y áreas conurbadas de Villa Allende, Saldán, etc. al Norte. Fuente: elaboración propia (2019) con base en datos de IDERA, DEYC, IPLAM y BAOTCba.

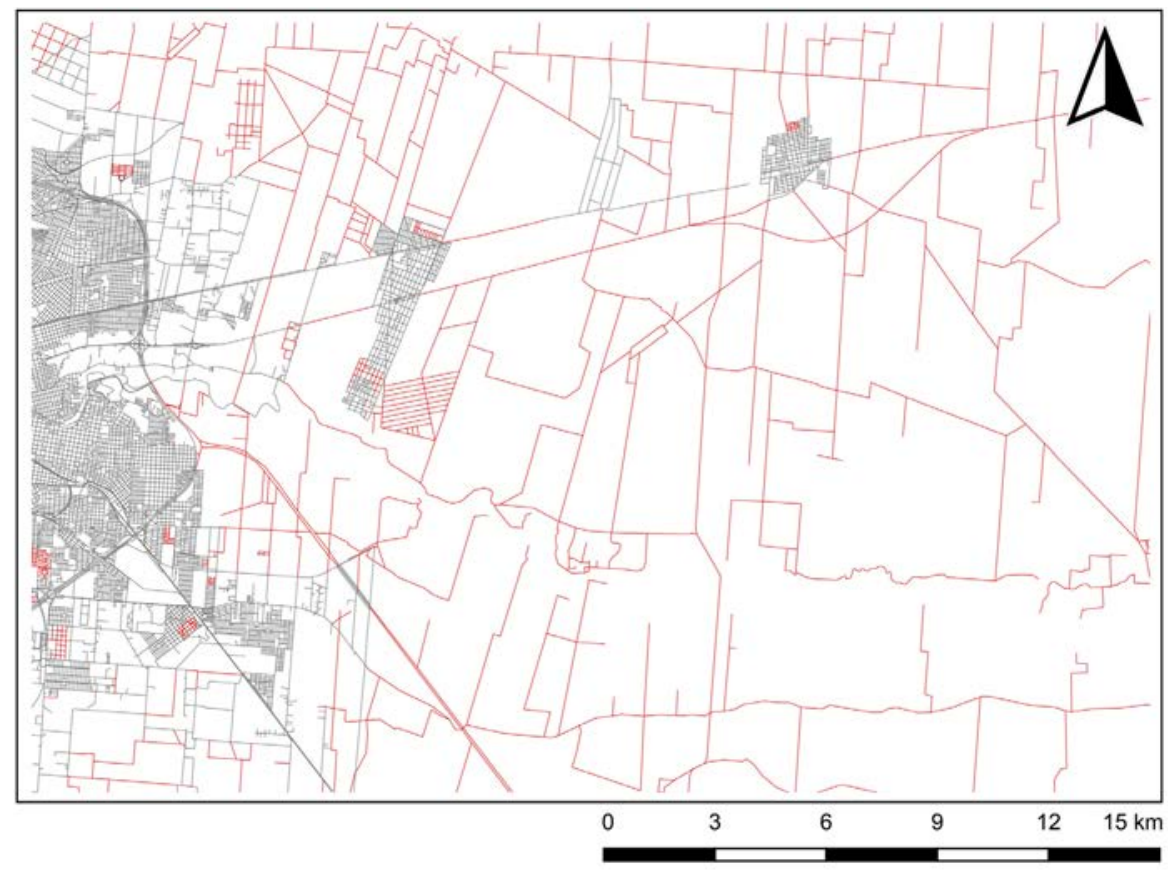

Figura 7. AMCBA. Red vial (2015) (detalle). Sector Este de Córdoba (izquierda) y áreas urbanizadas hacia el Este. Fuente: elaboración propia (2019) con base en datos de IDERA, DEyC, IPLAM y BAOTCba. 
El mapa axial se procesa con el complemento Space Syntax Toolkit para QGIS en combinación con el software depthmapX, ambos desarrollados por el Space Syntax Laboratory y de libre acceso. Como se adelantó, en primer lugar, se verifica el mapa axial, identificando y corrigiendo defectos de trazado. A continuación, se calculan con el paquete de software los valores de integración global y conectividad para cada uno de los vectores de la red. Los resultados obtenidos se muestran en las figuras 8 y 9 .

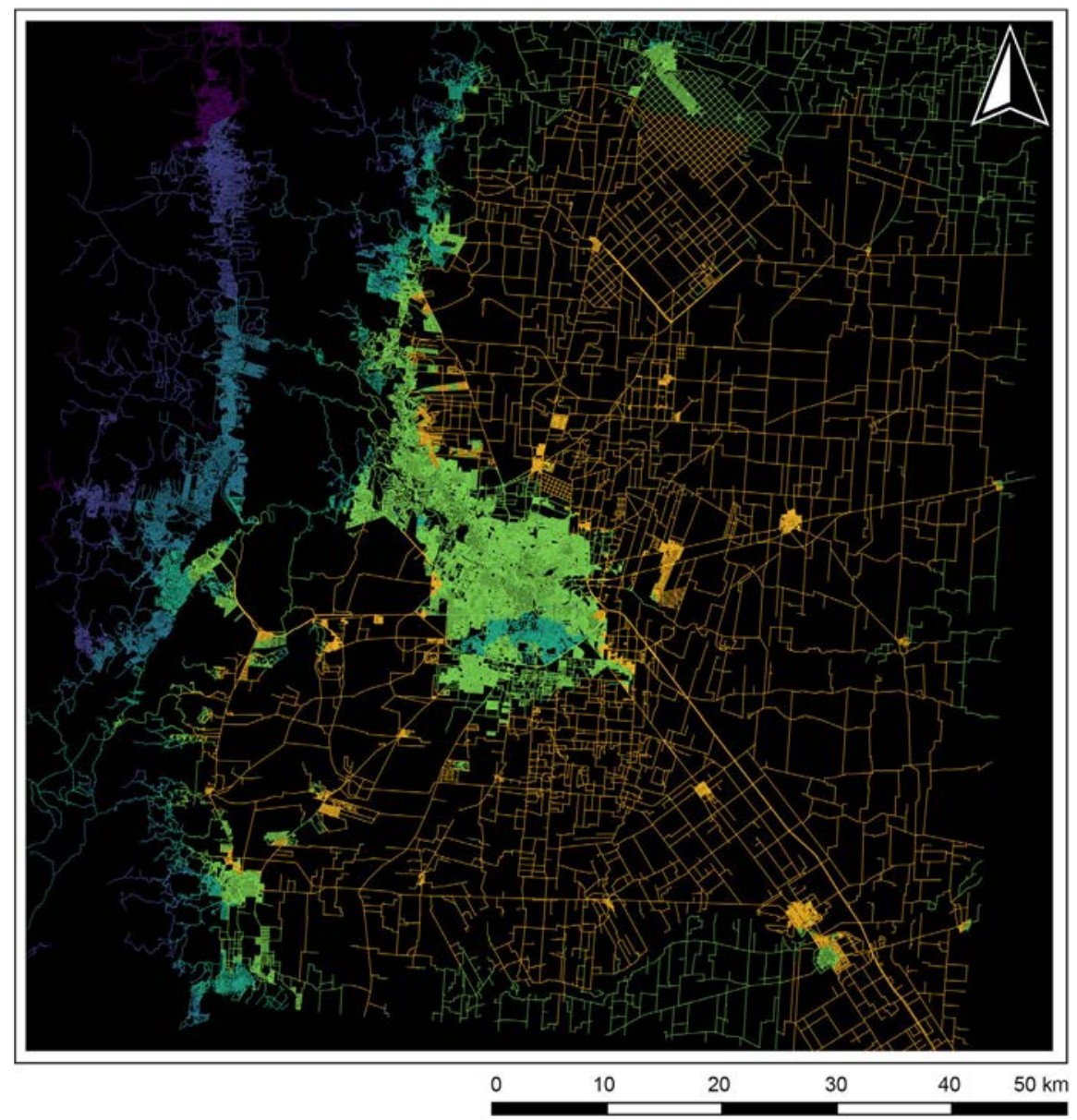

0,022 a 0,034 (1)

0,034 a 0,046 (2)

0,046 a 0,058

0,058 a $0,070 \quad(4)$

0,070 a 0,082

0,082 a 0,094

Figura 8. AMCBA. Red vial (2015) según niveles de integración global. La clasificación se define según intervalos iguales de los valores de la muestra; entre paréntesis se indica la valoración ordinal de acuerdo al nivel de integración global de las vías para cada clase. Fuente: elaboración propia (2019) con base en datos de BAOTCba, IPLAM y DGEyC, procesados con Space Syntax Toolkit para QGIS y dephmapX. 

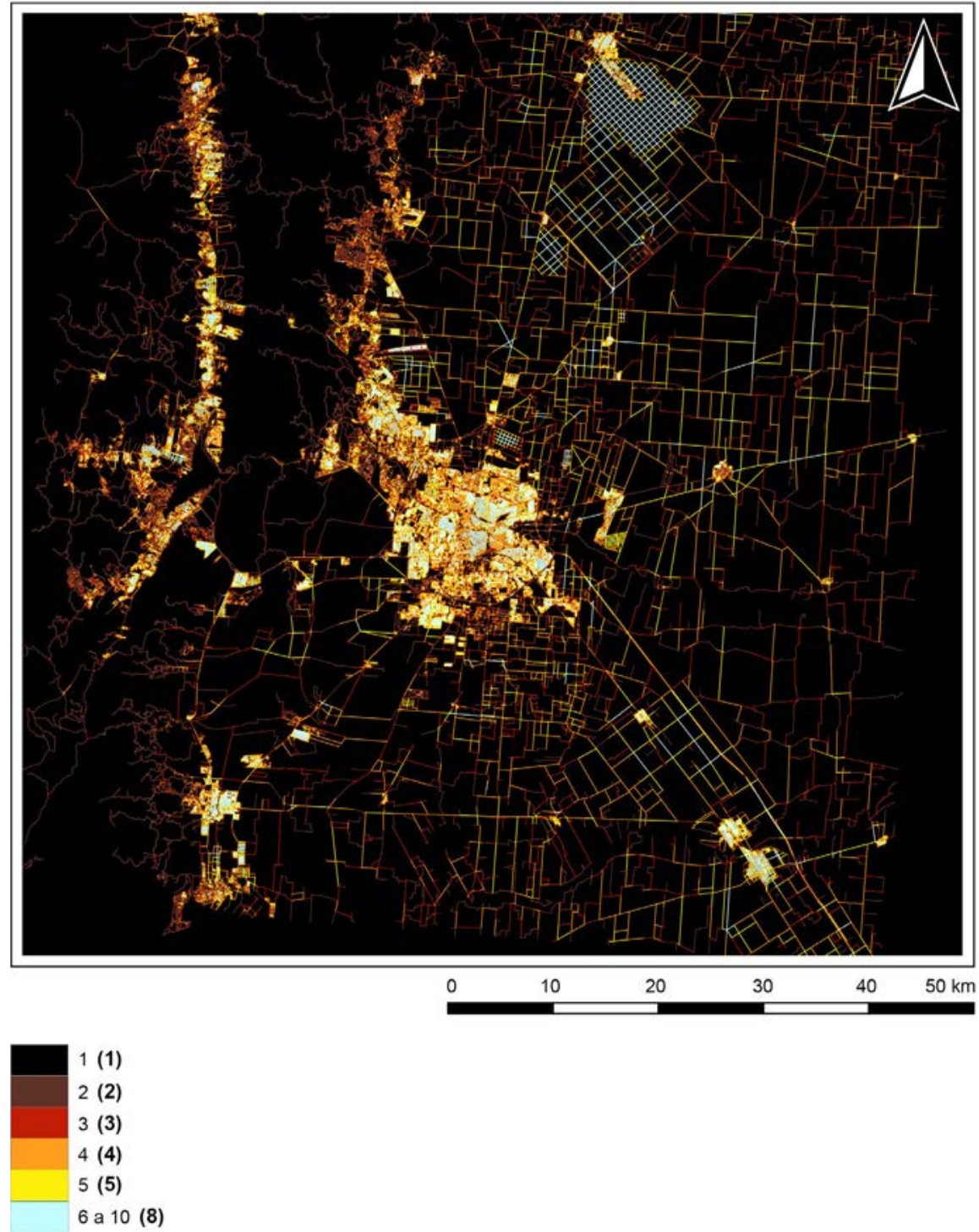

Figura 9. AMCBA. Red vial (2015) según niveles de conectividad. La clasificación se define según cortes naturales de los valores de la muestra; entre paréntesis se indica la valoración ordinal de acuerdo al nivel de conectividad de las vías para cada clase. Fuente: elaboración propia (2019) con base en datos de BAOTCba, IPLAM y DGEyC, procesados con Space Syntax Toolkit para QGIS y dephmapX.

Finalmente, para mejorar la lectura de los resultados obtenidos con el análisis de SE y profundizar el análisis de resultados, se combinan los valores de integración global y conectividad obtenidos para cada vector a través de la evaluación multicriterio (EMC), obteniendo un mapa de valoración integral para la red vial del AMCBA.

\section{Combinación de integración global y conectividad según una evaluación multicriterio}

La EMC es un instrumento metodológico que permite valorar cuali o cuantitativamente un fenómeno en función de diferentes criterios que son poco o nada semejantes, es decir, que no son directamente comparables entre si (Gómez Delgado y Barredo Cano, 2005:43). En este caso, es una herramienta que complementa el instrumental de la SE al posibilitar el análisis integrado de los dos indicadores que se exploraron individualmente, la integración global y la conectividad. 
Inicialmente, los resultados obtenidos para cada uno de los dos indicadores en el territorio analizado se clasifican según un método de valoración completa, agrupándolos en clases ordinales: los valores de integración global se agrupan en 6 clases según intervalos iguales de los valores de la muestra (Figura 8); los valores de conectividad se agrupan en 6 clases según cortes naturales en los valores de la muestra (Figura 9). Cada una de esas clases se pondera ordinalmente, desde un nivel mínimo a uno máximo ( 1 a 6 en el caso de la integración; en el caso de la conectividad, al sexto grupo se le asignó un valor de 8 debido a la mayor diferencia cuantitativa entre el quinto y el sexto grupo ${ }^{2}$. Esto permite que los niveles de los distintos indicadores sean valorados con clasificaciones homogéneas.

Para cada vector, los valores de cada indicador se combinan mediante una extensión jerárquica (concretamente, una suma ponderada): cada término de esta suma ponderada es un indicador afectado por un factor de ponderación en relación a su importancia para definir el atributo analizado (Gómez Delgado y Barredo Cano, 2005:62). Sin embargo, tanto para definir la clasificación como para definir la importancia relativa de cada indicador (es decir, el factor de ponderación que afectará a cada indicador en la suma ponderada) no hay métodos universalmente adoptados, ni parece haber consenso sobre este asunto en las investigaciones realizadas con este procedimiento (Gómez Delgado y Barredo Cano, 2005:77). El alto grado de discrecionalidad, tanto en esta etapa como en la anterior de reclasificación es uno de los puntos más discutidos de la EMC. Como criterio fundamental, el marco instrumental de la EMC requiere que las premisas seguidas en esta etapa sean sustentadas por el marco conceptual; esto, sumado a la transparencia en la definición de estos factores posibilita realizar procesos de validación y contrastabilidad para verificar su representatividad y fiabilidad; sin embargo, la SE no suele profundizar en una valoración integral de estos indicadores.

La EMC propone en estos casos el análisis de sensibilidad método de validación. Éste comprueba la estabilidad del método "examinando la variación que se produce en los resultados cuando se realizan variaciones sistemáticas en un rango de interés sobre uno o más parámetros de entrada" (Gómez Delgado y Barredo Cano, 2005:184). Este proceso permite determinar los indicadores que más influyen en el resultado, los menos significativos y los que interactúan con otros. El proceso implica una exploración por aproximaciones del resultado de combinar los valores de integración global y conectividad.

Para esto, primero, se revisan los resultados de combinar los valores clasificados de ambos indicadores sin afectarlos con factores de ponderación según muestra la ecuación (a); luego, se ensaya aumentar la importancia relativa de la integración global en relación a la conectividad (60\% a 40\%) como muestra la ecuación (b); por último, se ensaya aumentar la importancia relativa de la conectividad en relación a la conectividad (60\% a $40 \%)$ con la ecuación (c):

(a) Combinación simple $=I N T+C O N$

(b) Combinación(int) $=$ INT *0,60+CON*0,40

(c) Combinación(con) $=I N T * 0,40+C O N * 0,60$

Donde:

Combinación simple: valor combinado de los niveles de integración global y conectividad sin afectarlos con un factor de ponderación.

Combinación(int): valor combinado de los niveles de integración global y 
conectividad afectados con un factor de ponderación que aumenta la importancia relativa de la integración global.

Combinación(con): valor combinado de los niveles de integración global y conectividad afectados con un factor de ponderación que aumenta la importancia relativa de la conectividad.

INT: valor clasificado de la integración global.

CON: valor clasificado de la conectividad.

Las tres combinaciones se realizan con las herramientas de cálculo de campo de QGIS, editando la tabla de atributos de los vectores que componen el mapa axial. Al comparar la visualización de los resultados de las tres combinaciones, no se observan diferencias significativas. La ponderación relativa no se considera relevante y, por lo tanto, el análisis se realiza con los resultados de la combinación simple (a), que se muestran en la figura 10.

Los tres mapas obtenidos (Figuras 8, 9 y 10) permiten conocer los niveles de conectividad e integración que poseen las distintas partes de la red vial. Los resultados del análisis se desarrollan a continuación.
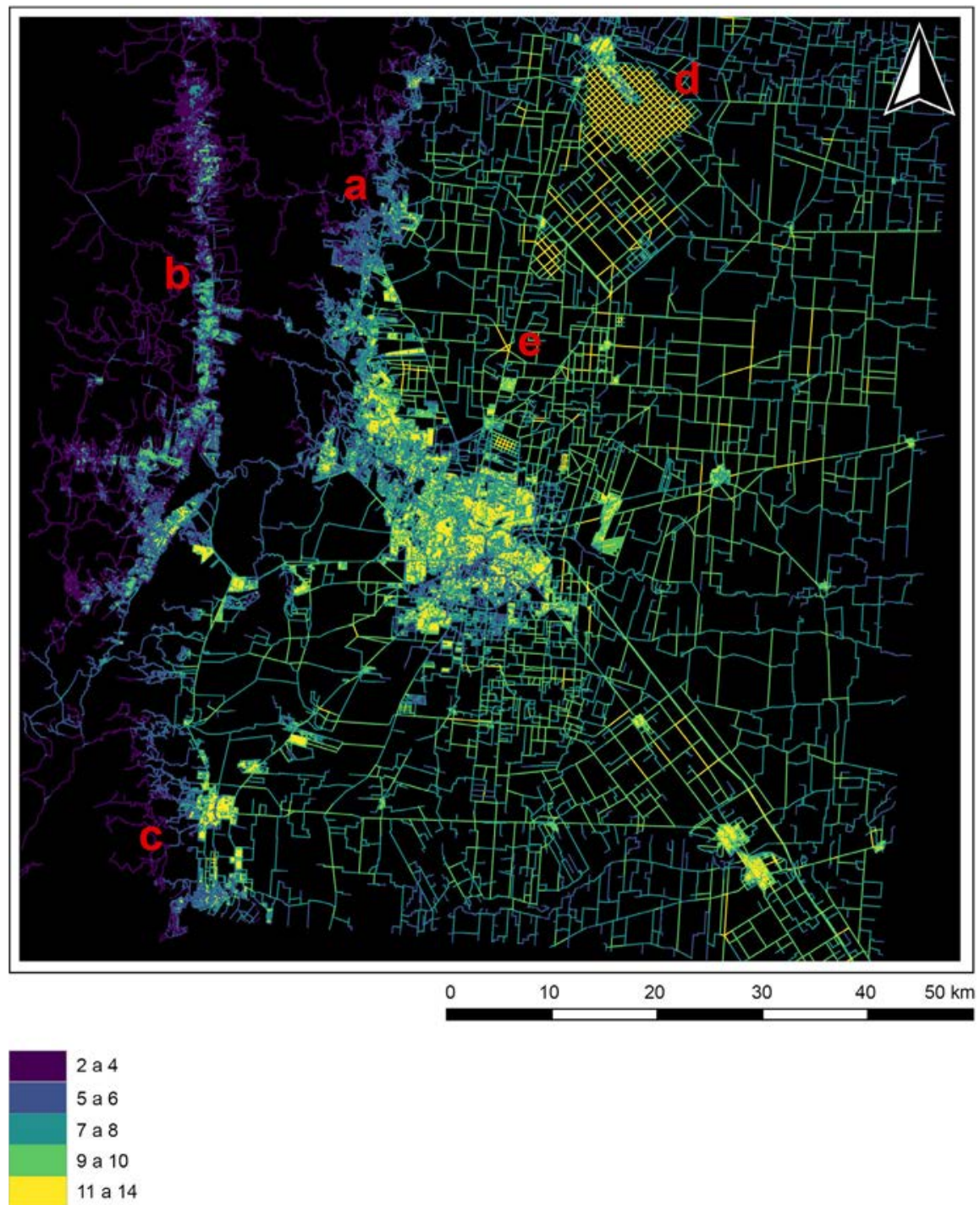

Figura 10: AMCBA. Red vial (2015) según niveles de integración global y conectividad combinados. La clasificación se define según cortes naturales de los valores de la muestra. Fuente: elaboración propia (2019) con base en datos de BAOTCba, IPLAM y DGEyC, procesados con Space Syntax Toolkit para QGIS y dephmapX. 


\section{Resultados del análisis}

El análisis preliminar tanto de las figuras 8 y 9 parece tan sólo confirmar hipótesis de trabajo consolidadas a lo largo de décadas de estudios regionales latinoamericanos y particularmente del AMCBA:

En las áreas centrales y pericentrales de las aglomeraciones urbanas se concentra la mayor densidad de vectores; estos vectores tienen, además, los niveles más altos de conectividad, al estar articulados en trazados tipo malla, o tipo retícula más o menos regular. En las áreas periurbanas y rurales, mientras tanto, la densidad de vectores disminuye; al mismo tiempo, disminuye también la cantidad de conexiones directas de cada vector ubicado en esas zonas ${ }^{3}$.

Los mapas muestran además un sistema altamente centralizado y fuertemente anisotrópico, incluso cuando se trata de áreas urbanizadas: la aglomeración principal (la ciudad de Córdoba) tiene niveles muy altos de conectividad e integración en el sistema, a diferencia de la red conurbada hacia el Noroeste a lo largo de la ruta E-53 (a), con niveles inferiores de integración y conectividad, o los aún menores niveles del corredor que se extiende en el Valle de Punilla tras las Sierras Chicas (b), o el territorio alrededor de Alta Gracia (c) (Figura 10).

La topografía serrana sobre la que se extiende el sector Oeste de la red vial, que dificulta el trazado de vías que vinculen estos corredores entre sí y con el resto de la red (siendo el cordón de las Sierras Chicas una evidente barrera entre el sector Oeste y el resto de la red, como se aprecia en los mapas obtenidos), explica en parte los bajos niveles de integración global y conectividad de ese sector. Sin embargo, el área Este de la red, que se extiende sobre la llanura pampeana -y que tiene niveles de integración altostiene niveles similares o menores de conectividad. Los aglomerados urbanos sobre la llanura Este están además aún más desconectados del resto de la red metropolitana, lo que podría explicarse por la estructura socio-productiva de esa área, dedicada casi exclusivamente a la producción agrícola extensiva cuyas unidades productivas ocupan grandes extensiones territoriales.

Por último, debe mencionarse que aún dentro del área urbanizada más consolidada de Córdoba (Boccolini, 2018) emergen sectores de gran conectividad interna, pero en parte segregados del resto del sistema. Aunque en esto intervienen, por supuesto, barreras naturales como el río Suquía y las barrancas al Norte y al Sur de su cauce, también las vías de conexión rápida como avenidas y autopistas fragmentan el tejido urbano. Estas vías, si bien permiten la integración entre sectores lejanos de la red, disminuyen la conectividad entre sectores adyacentes (Ver al respecto la discusión planteada en Greene y Mora, 2005). Esto es un reflejo explícito del proceso de urbanización por fragmentos que caracteriza a este tipo de regiones metropolitanas: esas urbanizaciones, aunque conurbadas eventualmente, difícilmente pueden aumentar su conectividad con los otros fragmentos del sistema. Tal como se planteó inicialmente, en un sistema urbano la estructura vial es uno de los elementos construidos con mayor inercia en su desarrollo a lo largo del tiempo.

Una vez revisados estos resultados, puede afirmarse que, al restringir la investigación al marco específico de la SE, el análisis -si bien conlleva etapas de reconstrucción y cálculo muy laboriosas- se muestra poco capaz de profundizar en premisas que ya se han planteado cualitativamente, basadas sólo en las características geométricas de la red 
vial. Sin embargo, los resultados en función de la SE obtenidos en este trabajo revelan su potencial cuando son contrastados con otras dimensiones de análisis territorial. Para ello, los mapas se comparan individualmente con los patrones de uso del suelo (Figura 2), distribución de niveles de densidad de población (Figura 11), distribución de EySU específicos (Figura 12), crecimiento/decrecimiento demográfico (Figura 13) y distribución de la población según su nivel socioeconómico (Figura 14).

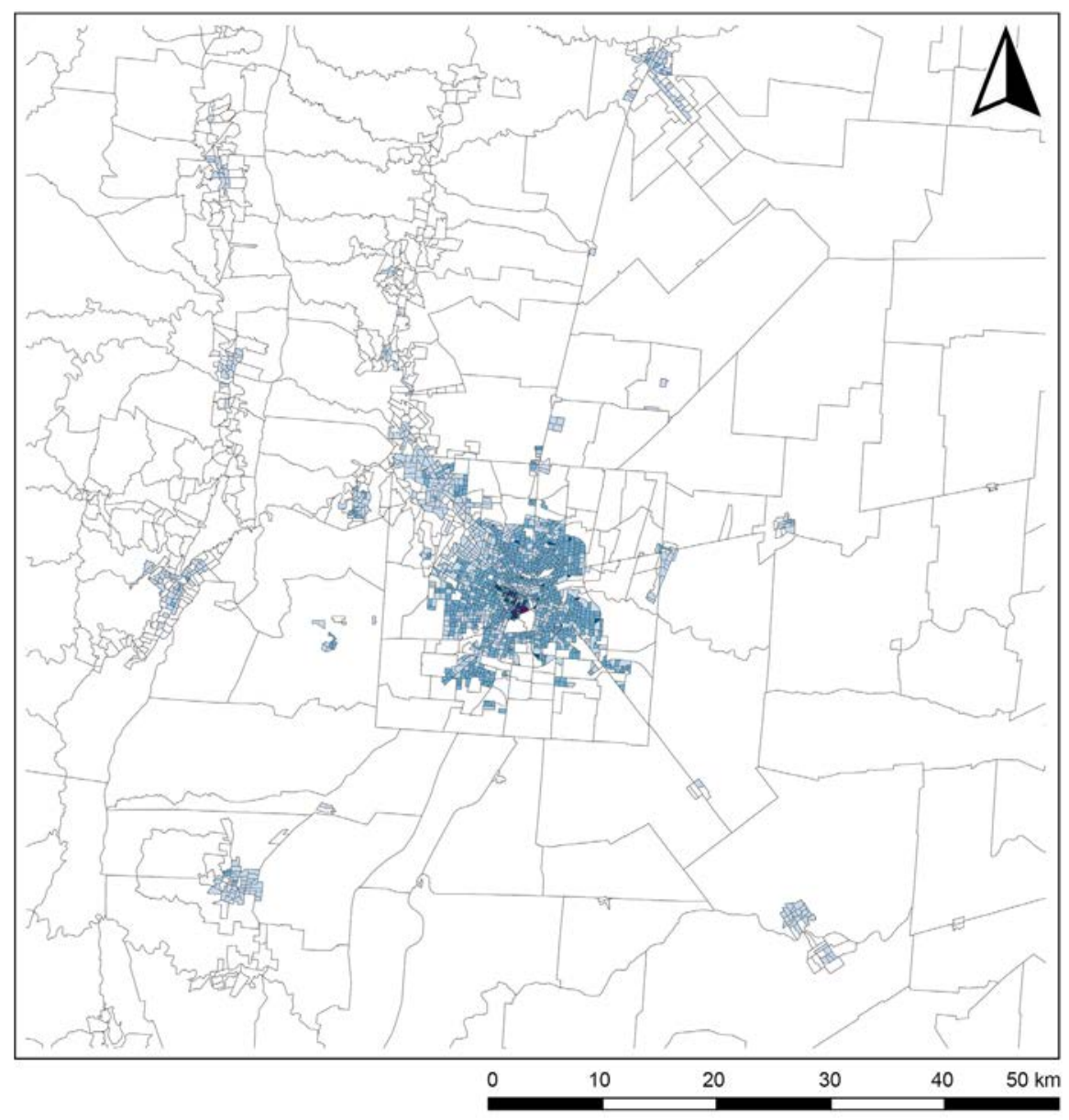

0 a 25

25 a 67

67 a 163

163 a 343

343 a 605

Figura 11. AMCBA. Densidad de población en cada radio censal (2010). Fuente: elaboración propia (2018) con base en datos del INDEC y de la DGEyC.

Si se compara la figura 10 (combinación simple) con la figura 11 (densidad de población), se observa que la población tiende a concentrarse en las áreas de mayor conectividad e integración: el $62 \%$ de la población metropolitana ( 1.158 .000 personas aproximadamente) vive allí. Estas áreas, además, albergan la mayor cantidad de EySU especializados (figura 12) a escala metropolitana, regional y provincial. 


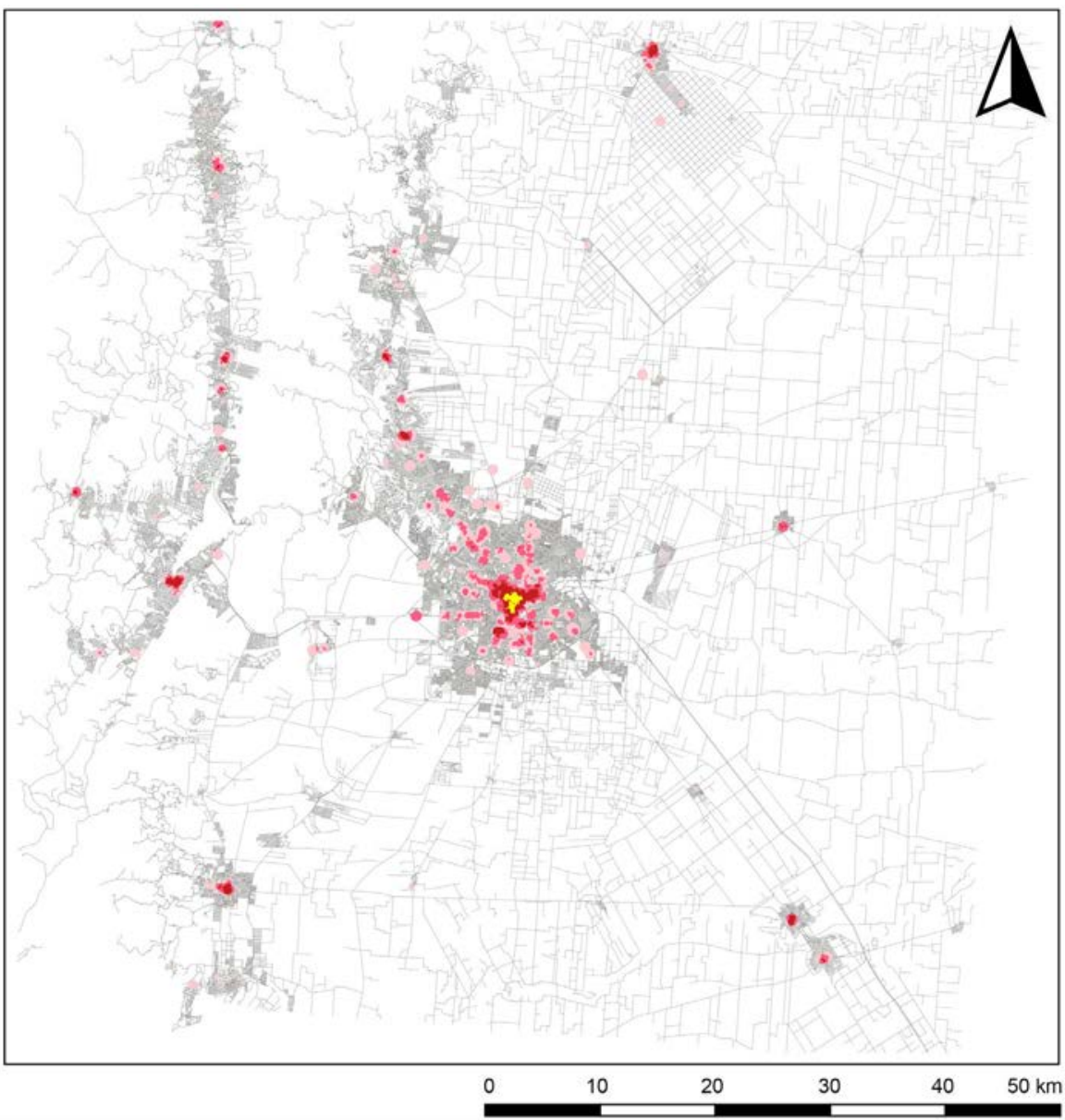

Nulo (0 a 8 )

\section{Bajo (9 a 15)}

Medio (16 a 23)

Alto (24 a 40)

Muy alto (41 a 57$)$

Figura 12. AMCBA. Disponibilidad de equipamiento y servicios urbanos específicos (2018). El mapa muestra, además, la división en radios censales (Censo Nacional 2010) como referencia. Fuente: elaboración propia (2018) con base en datos de IDERA, proveedor de data GIS (Garmin), Google Maps (www.maps.google.com.ar), la DGEyC y la base de datos del Consejo Nacional de Investigaciones Científicas y Técnicas (CONICET).

Esto podría conducir a afirmar que la alta concentración vial se corresponde con la concentración de población y EySU en el territorio: a pesar de su macrocefalia, y aún cuando el área periurbana y rural tienen graves deficiencias en conectividad e integración global, la mayor parte de la población urbana se localiza en áreas con muy buena accesibilidad a los EySU disponibles en la región. 


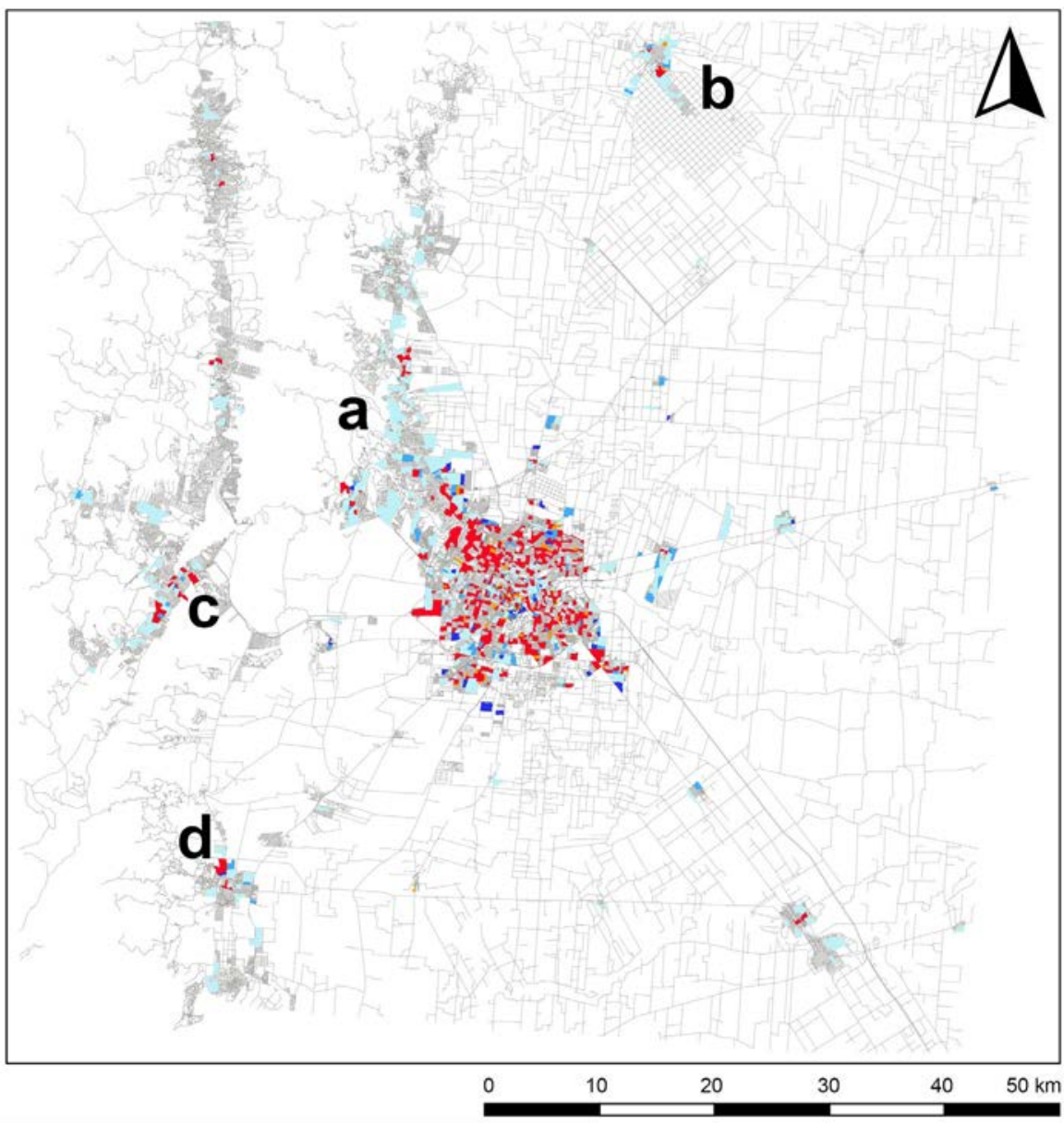

Disminución de entre 20 y 53 hab/ha

Disminución de entre 5 y 20 hab/ha

Variación no significativa (entre $-5 y+5$ hab/ha)

Aumento de entre 5 y 20 hab/ha

Aumento de entre 20 y 50 hab/ha

Aumento de entre 50 y $104 \mathrm{hab} / \mathrm{ha}$

Figura 13. AMCBA. Diferencia intercensal de la densidad de población (2001-2010). Fuente: elaboración propia (2019) con base en datos censales georreferenciados de la DEyC y mapas de radios censales 2001 y 2010 trazados por Gonzalo Rodríguez (CEUR-CONICET).

Sin embargo, al analizar históricamente las tendencias de crecimiento y redistribución demográficas (figura 13) se descubre que las áreas centrales de los principales aglomerados urbanos -Córdoba, Villa Allende (a), Colonia Caroya (b), Carlos Paz (c), Alta Gracia (d)- y gran parte del área pericentral de Córdoba reducen su población desde al menos 20 años (según datos de la Dirección de Estadística y Censos de la Provincia), lo que implica que las áreas mejor servidas y con mayor accesibilidad de la región están reduciendo notablemente su población. Al mismo tiempo, las mayores tasas de crecimiento demográfico emergen en áreas periurbanas de los grandes aglomerados y en áreas urbanas y periurbanas de aglomerados medianos y pequeños alrededor de la ciudad principal.

Las causas de este proceso de contraurbanización son variadas -motivos económicos, de estilo de vida, oferta habitacional disponible, mejor calidad ambiental en áreas periurbanas y rurales, entre otros- (Boccolini, 2018; Peralta y Liborio, 2014b; Tecco, 
1999), pero convergen en un proceso de crecimiento demográfico en áreas con bajos niveles de integración y conectividad que se traduce en bajos niveles de accesibilidad a EySU (figura 12). Esto se agrava al considerar que esas áreas, sobre todo las ubicadas al Noroeste de Córdoba y en el Valle de Punilla, presentarán las mayores dificultades (en cuanto a proyecto y ejecución) y costos (financieros, pero también ambientales ${ }^{4} \mathrm{y}$ sociales) para mejorar sus niveles de integración y accesibilidad vial.

Esta tendencia de crecimiento demográfico hacia el sector serrano del AMCBA y la dificultad planteada por la propia topografía para mejorar su integración, podrían motivar que el crecimiento demográfico -y, por supuesto, el mayor interés de inversores y desarrollistas inmobiliarios- se oriente a consolidar y expandir el área urbanizada a lo largo del corredor de la ruta E53 al Noroeste de Córdoba -casi hasta llegar a la Ruta 9 al Norte (e)-, y sobre las rutas 5 y C45 al Suroeste, hasta Alta Gracia y las localidades menores ubicadas al Sur, donde los niveles de accesibilidad actuales son mayores, y la topografía de piedemonte -es decir, de transición entre la sierra y la pampa- presenta menos obstáculos para la ejecución de obras de mejoramiento y extensión de la red vial.

Es necesario mencionar que, ya que esos sectores serranos suman ventajas de localización y accesibilidad, es probable que allí se concentren especialmente los proyectos privados de desarrollo urbano a corto y mediano plazo. Teniendo en cuenta que estos proyectos inmobiliarios privados tienden a focalizarse en la producción habitacional destinada a los usuarios (e inversores) con mayor capacidad de pago (Boccolini, 2017a; Capdevielle, 2014b), eso podría conducir a la consolidación de esos territorios como residencia de la población de mayor NSE, incluso expulsando eventualmente a sus actuales habitantes de menor NSE. Mientras tanto, los proyectos de vivienda social destinada a las personas de menor NSE tienden a ubicarse en áreas rurales y periurbanas con niveles medios a bajos de integración y muy bajos de accesibilidad (Boccolini, 2017b; Elorza, 2016; Capdevielle, 2014a; Tecco y Fernández, 2005). Con esto, las de mejor accesibilidad urbana y calidad ambiental del AMCBA -después de las áreas urbanas centrales de los grandes aglomerados-, se transformarán en territorios altamente segregados socioeconómicamente, ocupados casi exclusivamente por la población de mayor NSE.

Estos patrones de polarización de la población están ya consolidados en el territorio metropolitano, tal como señala la Figura 14, que muestra los niveles de segregación residencial socioeconómica en el momento del último censo nacional en 2010. Sin embargo, las condiciones arriba mencionadas hacen que sea posible prever una aceleración en la tendencia de polarización de la población de acuerdo a su NSE a escala metropolitana a mediano y largo plazo. 


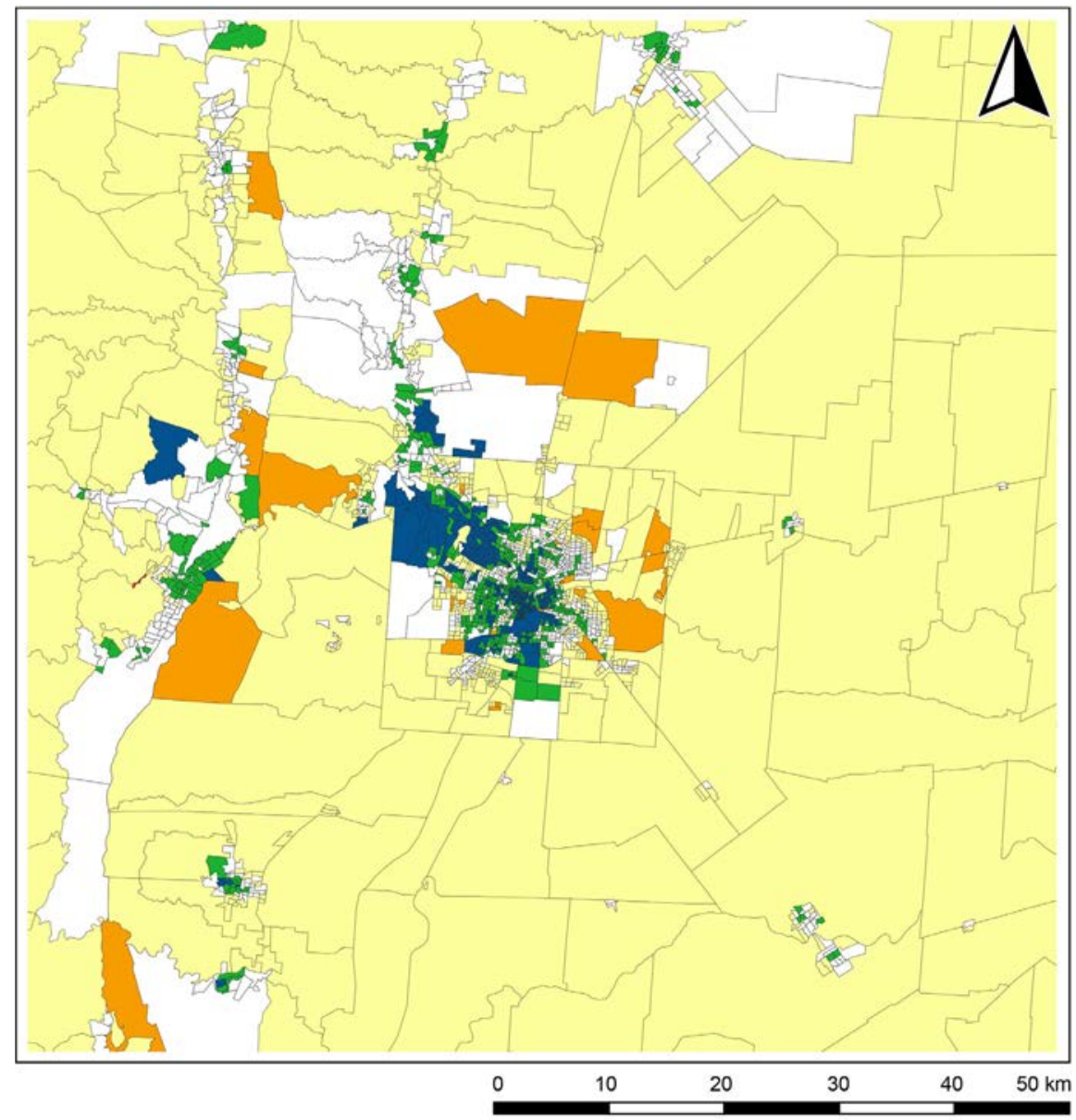

SRS Muy alta (predominio de hogares con BAJO NSE)

SRS Alta (predominio de hogares con BAJO NSE) SRS Baja (predominio de hogares con BAJO NSE) SRS Nula

SRS Baja (predominio de hogares con ALTO NSE)

SRS Alta (predominio de hogares con ALTO NSE)

Figura 14. AMCBA. Segregación residencial socioeconómica en cada radio censal, en función de la población provincial (2010). Fuente: elaboración propia (2018) con base en datos del INDEC y de la DGEyC.

Comparar las Figuras 8 y 9 con la Figura 14 muestra, además, otros efectos de esta polarización socioeconómica en el territorio. La población de alto NSE, y alta SRS tienden a localizarse en sectores urbanos que, si bien tienen altos niveles de integración global, tienen bajos niveles de conectividad con los elementos adyacentes de la red vial. El ejemplo más notorio son las áreas intermedias y periurbanas al Sur y las Noroeste de Córdoba (Figura 10), con niveles de integración y conectividad mucho más bajos que las áreas urbanizadas adyacentes, y que coinciden con las áreas donde reside casi excluyentemente la población de más alto NSE. Estas áreas, además, suelen ser las únicas que cuentan con un nivel medio o alto de EySU además de las áreas centrales de los principales aglomerados urbanos.

Coincidiendo con otros estudios que arriban a estos resultados (Tagliari, 2018; Tibiriçá de Saboya et al., 2016; Silva, Loch y Da Cruz Silva, 2009), puede sostenerse que la población de mayor NSE tiende a ubicarse en sectores urbanos que, aún disponiendo 
de una muy buena provisión de EySU (Figura 12), tienen una conectividad baja o muy baja con el resto del territorio, sobre todo las áreas adyacentes donde reside la población de menor NSE.

Por otro lado, los sectores donde reside la población con bajo NSE más segregada en el territorio son lugares con poca presencia de la red vial, y cuyas escasas vías tienen niveles muy bajos de conectividad con el resto del AMCBA, a pesar de contar con muy buena integración global (Figura 14 y 10). Si se tiene en cuenta la gran concentración de EySU en áreas urbanas centrales y de alto NSE (Figuras 14 y 12), esto supone que, para esta población, es más factible acceder a EySU que encuentran en las alejadas áreas mejor servidas de la región que encontrar EySU cercanos a su residencia: a pesar de la mayor distancia, es más fácil trasladarse a las distantes áreas centrales y pericentrales de los mayores aglomerados urbanos que a los vecinos sectores urbanos -periferias o pequeños aglomerados urbanos próximos a su ubicación.

Incluso sin profundizar en la materialidad, espacialidad o capacidad de la red vial en esos sectores, lo anterior no sólo muestra la escasa posibilidad de que estas regiones mejoren su calidad urbana, sino también un aspecto más de la gran dependencia de la población de EySU altamente concentrados en el territorio. Al tratarse de población con bajo o muy bajo NSE, la exclusión de las áreas mejor servidas de la región no sólo se debe a la gran distancia y escasez de vías alternativas de conexión, sino también al mayor costo (económico, de tiempo, de sobrecarga a miembros a cargo de la supervisión de otros familiares) que significa el traslado periódico a esos lugares, y que deben afrontar las personas con los ingresos familiares más bajos de la región metropolitana.

Estos resultados apoyan la hipótesis de que las áreas donde habita la población de alto NSE acaparan EySU en desmedro de áreas donde reside la población de NSE medio bajo o bajo, junto con las "oportunidades diferenciales de acceso a toda clase de recursos que tal condición define" (Groisman, 2010: 342). Eso, sumado a las tendencias de redistribución poblacional (contraurbanización, periurbanización, vaciamiento de áreas centrales) muestra la urgencia de replantear el modelo macrocefálico de organización metropolitana y el rol de la red vial como posibilitante o como obstáculo para avanzar hacia un desarrollo regional más inclusivo y equitativo.

El análisis realizado en este trabajo confirma que la SE es un instrumento con gran potencial para comprender el desarrollo territorial de una región metropolitana. El enfoque aplicado, además, plantea un aporte en la comprensión del desarrollo del sistema metropolitano como un proceso dialéctico entre las diferentes dimensiones que lo estructuran: retomando la premisa de análisis planteada en la presentación de este trabajo, no es posible realizar un análisis crítico de la red vial sólo con las herramientas analíticas que ofrece la SE. Lo que la SE ofrece es una descripción de la capacidad de la red vial regional para conducir los flujos de personas y bienes materiales en la región metropolitana. En este caso concreto, la información obtenida específicamente del análisis de la SE tiene poco poder explicativo por sí misma, y es necesario incorporar otras dimensiones de análisis para comprender las implicancias de los niveles de integración y conectividad en el sistema metropolitano. Sin embargo, combinando los resultados con análisis complementarios -tal como se propone en este trabajo-, los resultados ganan en profundidad de análisis y capacidad explicativa.

Esto abre el camino para estudios que combinen el análisis por medio de la SE con estudios perceptuales, socioculturales, políticos y económico-productivos, pero también de estudios espaciales en cuanto envolventes y caudales de los elementos de la red, sirviéndose de sus resultados para explicar distintos aspectos del desarrollo espacial y humano a escala regional. 


\section{Siglas utilizadas}

AMCBA: área metropolitana de Córdoba (región metropolitana de Córdoba).

BAOTCba: Bases Ambientales para el Ordenamiento Territorial del Espacio Rural

de la Provincia de Córdoba

CONICET: Consejo Nacional de Investigaciones Científicas y Técnicas

DEyC: Dirección Provincial de Estadística y Censos de Córdoba

EMC: evaluación multicriterio

EySU: equipamiento y servicios urbanos

IPLAM: Instituto de Planificación del Área Metropolitana

NSE: nivel socioeconómico

SE: Sintaxis espacial 


\section{Q Bibliografía}

» ADEC (Agencia para el desarrollo económico de Córdoba). (2014). Proyecto: Prospectiva Territorial para el Área Metropolitana de Córdoba [Informe descriptivo]. Córdoba: ADEC - Municipalidad de Córdoba. https://goo.gl/ WK6NHR

» ADEC (Agencia para el desarrollo económico de Córdoba). (2015a). Proyecto: Prospectiva Territorial para el Área Metropolitana de Córdoba. Escenarios [Informe descriptivo]. Córdoba: ADEC - Municipalidad de Córdoba. https:// goo.gl/WK6NHR

» ADEC (Agencia para el desarrollo económico de Córdoba). (2015b). Proyecto: Prospectiva Territorial para el Área Metropolitana de Córdoba. Interrogantes e hipótesis [Informe descriptivo]. Córdoba: ADEC - Municipalidad de Córdoba. https://goo.gl/WK6NHR

" Batty, M. (2001). Agent-based pedestrian modeling. Environment and Planning B: Planning and Design, (XXVIII), 321-326. DOI: 10.1068/b2803ed

" Boccolini, S. (2017a). Producción privada de hábitat urbano en Córdoba, Argentina: condiciones internas y contextuales que organizan su desarrollo. Papeles de Geografía, (63), 161-180. https://goo.gl/Su8ZPc

" Boccolini, S. (2017b). Impacto de políticas de vivienda en acceso al hábitat urbano en Córdoba, Argentina. Revista de Urbanismo, (37), 1-18. http://www. revistaurbanismo.uchile.cl/index.php/RU/article/view/46198

» Boccolini,S.(2018). Regulação urbana em Córdoba. ¿para uma cidade sustentável e inclusiva? Urbe, X(supl.1), 96-117. DOI: dx.doi.org/10.1590/2175-3369.010. supl1.a0o6

"Capdevielle, J. (2014a). Espacio urbano y desigualdades: las políticas públicas y privadas en la ciudad de Córdoba, Argentina (1990- 2011). Cuadernos Geográficos, (53)2, 135-158. https://www.redalyc.org/html/171/17132953006/

"Capdevielle, J. (2014b). Los grupos "desarrollistas» y su incidencia en el espacio urbano de la ciudad de Córdoba, Argentina (1990- 2013). Terra Nueva Etapa, $\quad(X X X) 47, \quad$ 129-152. http://www.scielo.org.ve/scielo.php?pid=S101270892014000100007\&script=sci_arttext\&tlng=es

»Dirección de Planeamiento urbano - Secretaría de Planeamiento Urbano Dirección de Hábitat. (1981). PlanDeMet: Plan de Desarrollo Metropolitano de Córdoba. Documento síntesis. Córdoba: Municipalidad de Córdoba.

"Elorza, A. L. (2016). Segregación residencial socioeconómica y la política pública de vivienda social el caso de la ciudad de Córdoba (Argentina). Cuaderno Urbano, 20(20), 71-94. http://arq.unne.edu.ar/publicaciones/cuaderno_urbano/cu_20/ archivos/pdf/elorza.pdf

» Gómez Delgado, M. y Barredo Cano, J. (2005). Sistemas de información geográfica y evaluación multicriterio en la ordenación del territorio. Madrid: RA-MA.

" Greene, M. y Mora, R. (2005). Las autopistas urbanas concesionadas: Una nueva forma de segregación. ARQ(Santiago), (60), 56-58. DOI: 10.4067/So71769962005006000009

»Groisman, F. (2010). La persistencia de la segregación residencial 
socioeconómica en Argentina. Estudios Demográficos y Urbanos, XXV(2 (74)), 429-46o. www.jstor.org/stable/20787558

"Hanson, J. (2003). Decoding Homes and Houses. Londres: Cambridge University Press.

" Hillier, B. (2015). Space Is the Machine: A Configurational Theory of Architecture. Londres: Space Syntax.

» Hillier, B. y Julienne, H. (2014). The Social Logic of Space. Londres: Cambridge University Press.

" IPLAM. (2017). Lineamientos para un sistema de transporte público de pasajeros [Documento de trabajo]. Córdoba: IPLAM - Gobierno de la Provincia de Córdoba.

"Irós, G. M., Moiso, E. A., Bravo, A. O., Alonso, C. S. y Gómez, L. (Eds.). (2012). Lineamientos del plan estratégico urbano territorial de la región metropolitana de Córdoba. Córdoba: Ministerio de Infraestructura de la Provincia de Córdoba Instituto de Planificación del Área.

"Peralta, C. y Liborio, M. (2014a). Redistribución poblacional en la ciudad de Córdoba entre los períodos intercensales 1991-2001 / 2001- 2008. Evaluación de los procesos de dispersión, densificación, gentrificación y renovación. Revista Vivienda y Ciudad, (1), 99-113. http://revistas.unc.edu.ar/index.php/ ReViyCi/article/view/9544

»Peralta, C. y Liborio, M. (2014b). Procesos de urbanización y desurbanización de los barrios pericentrales de la ciudad de Córdoba. En Primer Encuentro de Investigadores que Estudian la Ciudad de Córdoba. Realidad y ficción sobre la transferencia de las problemáticas urbanas predominantes, Córdoba, Argentina. https://cordobaedu.files.wordpress.com/2014/06/1c2ba-encuentro-deinvestigadores-que-estudian-la-ciudad-de-cc3b3rdoba.pdf

" Ratti, C. (2004). Space syntax: some inconsistencies. Environment and Planning B: Planning and Design, XXI, 487-499. DOI:10.1068/b3019

"Scheel, A. Z., Mann, R. B. y Pavié, S. N. (2017). Expansión periférica y procesos de centralidad en Valdivia entre 1900-2015: un análisis desde la perspectiva de la sintaxis del espacio. AUS [Arquitectura / Urbanismo / Sustentabilidad], (19), 24-30. http://revistas.uach.cl/index.php/aus/article/view/55

"Silva, J. M., Loch, C. y Da Cruz Silva, S. (2009). A sintaxe espacial de Curitiba. Revista Brasileira de Cartografia, LXI(2), 153-163. http://www.seer.ufu.br/index. $\mathrm{php} /$ revistabrasileiracartografia/article/view/44845

»Stavroulaki, G., Marcus, L., Berghauser Pont, M. y Staffan Nilsson, L. (2017). Representations of Street Networks in Space Syntax: Towards flexible maps and multiple graphs. En 11th Space Syntax Symposium, Lisboa, Portugal. https:// research.chalmers.se/publication/251296/file/251296_Fulltext.pdf

" Tagliari, V. (2018). Un análisis de sintaxis espacial de la estructura de Santa Fe. En Encuentro de jóvenes investigadores, Santa Fe, Argentina. http://bibliotecavirtual. unl.edu.ar:808o/colecciones/bitstream/handle/123456789/8871/RAD25.pdf

» Tecco, C. (1999). Periurbanización y metropolización, desafíos y cuestiones críticas en el Área Metropolitana Córdoba. Administración pública y sociedad, (12), s/d.

» Tecco, C., \& Fernández, S. (2005). Políticas públicas y segregación residencial socioeconómica en la ciudad de Córdoba, Argentina. En VII Seminario Nacional de la Red de Centros Académicos para el Estudio de Gobiernos Locales, Buenos 
Aires, Argentina.

» Telega, A. (2016). Urban Street Network Analysis Using Space Syntax in GIS - Cracow Case Study. En Baltic Geodetic Congress (BGC Geomatics), Gdansk, Polonia. DOI: 10.1109/bgc.geomatics.2016.57

» Tibiriçá de Saboya, R., Reis, A. F. y Portilho Bueno, A. (2016). Continuidades e descontinuidades urbanas à beira-mar: uma leitura morfológica e configuracional da área conurbada de Florianópolis. Oculum Ensaios, 13(1), 129152. http://www.redalyc.org/resumen.oa?id=351747444007

" UVITEC (Unidad de Vinculación Tecnológica Córdoba) e Innovos Group SA. (2017). Necesidades, oferta y demanda de servicios tecnológicos en Córdoba y su área metropolitana [Informe descriptivo]. Córdoba: ADEC - Municipalidad de Córdoba. https://goo.gl/WK6NHR

\section{Sara M. Boccolini / saraboccolini@gmail.com}

Arquitecta (Universidad Nacional de Córdoba-UNC, 2006); Magíster en Gestión y Desarrollo Habitacional (UNC, 2013); Doctora en Estudios Urbanos y Regionales (UNC y Bauhaus Universität Weimar, 2018). Investigadora Asistente de CONICET. Sus líneas de trabajo son la interfase periurbana de regiones metropolitanas medias y transformaciones de áreas urbanas consolidadas. Actualmente es parte del Centro de Investigaciones y Estudios sobre Cultura y Sociedad (CONICET-UNC) donde desarrolla el área de Estudios Regionales y Urbanos. 